Acta Crystallographica Section E

Structure Reports

Online

ISSN 1600-5368

\section{Andrei V. Churakov, ${ }^{a *}$ Lyudmila G. Kuz'mina, ${ }^{a}$ Petr V. Prikhodchenko ${ }^{\mathrm{a}}$ and Judith A. K. Howard ${ }^{b}$}

${ }^{\mathbf{a}} \mathrm{N}$. S. Kurnakov Institute of General and Inorganic Chemistry, Russian Academy of Science, 31 Leninskii prospect, Moscow 119991, Russian Federation, and bepartment of Chemistry, University of Durham, Science Laboratories, South Road, Durham DH1 3LE, England

Correspondence e-mail: churakov@igic.ras.ru

\section{Key indicators}

Single-crystal X-ray study

$T=120 \mathrm{~K}$

Mean $\sigma(\mathrm{C}-\mathrm{C})=0.002 \AA$

$R$ factor $=0.037$

$w R$ factor $=0.090$

Data-to-parameter ratio $=12.7$

For details of how these key indicators were automatically derived from the article, see http://journals.iucr.org/e.
(C) 2006 International Union of Crystallography All rights reserved

\title{
The first molecular structure containing four hydroperoxo groups: piperazine- 2,3,5,6-tetrayl tetrahydroperoxide pyrazine disolvate dihydrate
}

The reaction of pyrazine with hydrogen peroxide resulted in piperazine-2,3,5,6-tetrayl tetrahydroperoxide, crystallizing as its pyrazine disolvate dihydrate, $\mathrm{C}_{4} \mathrm{H}_{10} \mathrm{~N}_{2} \mathrm{O}_{8} \cdot 2 \mathrm{C}_{4} \mathrm{H}_{4} \mathrm{~N}_{2} \cdot 2 \mathrm{H}_{2} \mathrm{O}$. In the crystal structure, the tetraperoxo molecules, which possess a crystallographically imposed centre of symmetry, are linked into a three-dimensional network by hydrogen-bonding interactions involving the pyrazine and water molecules.

\section{Comment}

Peroxo derivatives of organic compounds attract particular interest as environmentally friendly bleaching compounds and oxidation agents in organic synthesis (Marwah et al., 2004). As part of our study of organic hydrogen peroxide solvates (Churakov et al., 2004, 2005), we tried to investigate the behaviour of small organic donor molecules, such as pyrazine or pyrimidine, in concentrated $\mathrm{H}_{2} \mathrm{O}_{2}$ solutions. The unexpected title compound, (I), was formed upon freezing of a pyrazine solution in $50 \%$ hydrogen peroxide. The nature of this process remains unclear. The centrosymmetric molecule of (I) (Fig. 1) contains four hydroperoxo substituents. The piperazine ring adopts a chair conformation and all hydroperoxo groups occupy axial positions. Atom N3 is slightly flattened, the sum of valence angles around it being $346.0^{\circ}$. The $\mathrm{O}-\mathrm{O}$ bond lengths [1.470 (1) and $1.471(1) \AA]$ are somewhat longer than the mean value of $1.462 \AA$ found for related compounds (85 entries, 106 fragments) in the Cambridge Structural Database (CSD, Version 5.27 of January 2006; Allen, 2002).

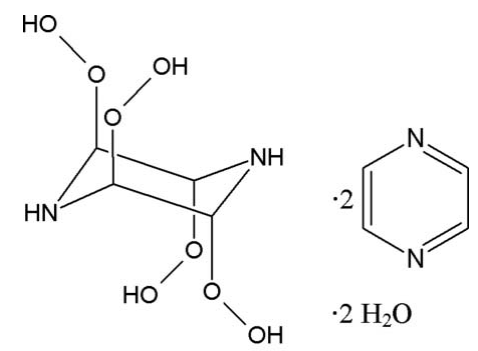

(I)

The hydroperoxo atom O3 acts as both donor (for symmetry-related) molecules and acceptor (for water molecules) of hydrogen bonds, forming layers perpendicular to the $c$ axis (Fig. 2). Pyrazine molecules accept hydrogen bonds from the peroxo $\mathrm{O} 4$ and water $\mathrm{O} 5$ atoms, cross-linking the layers of the main molecules into a three-dimensional network (Fig. 3).

To date, the CSD contains structures of compounds with no more than two hydroperoxo groups. The title compound is the
Received 21 April 2006 Accepted 4 May 2006 


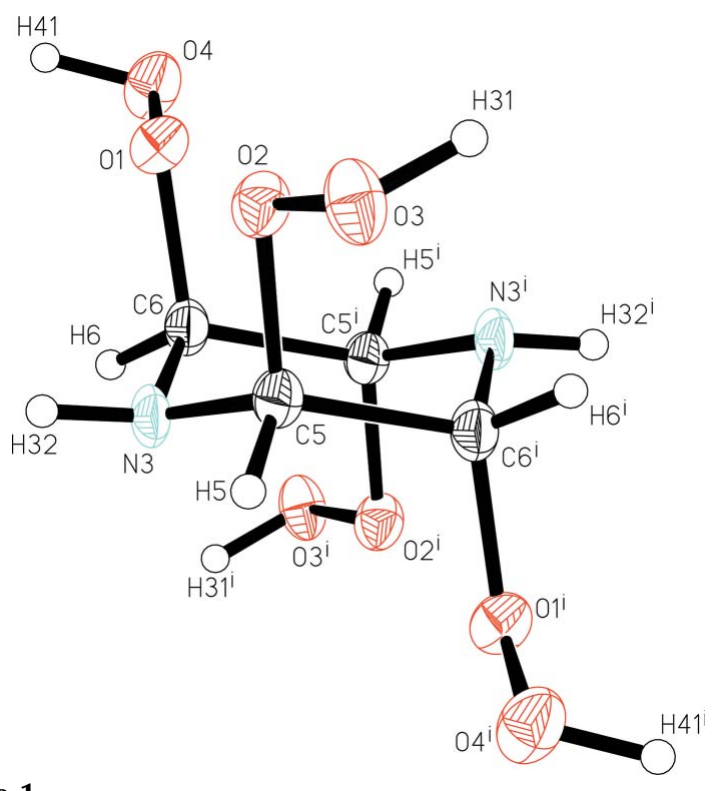

Figure 1

The molecular structure of the tetraperoxo molecule of (I), showing $50 \%$ probability displacement ellipsoids [symmetry code: (i) $1-y, 2-x$, $2-z]$.

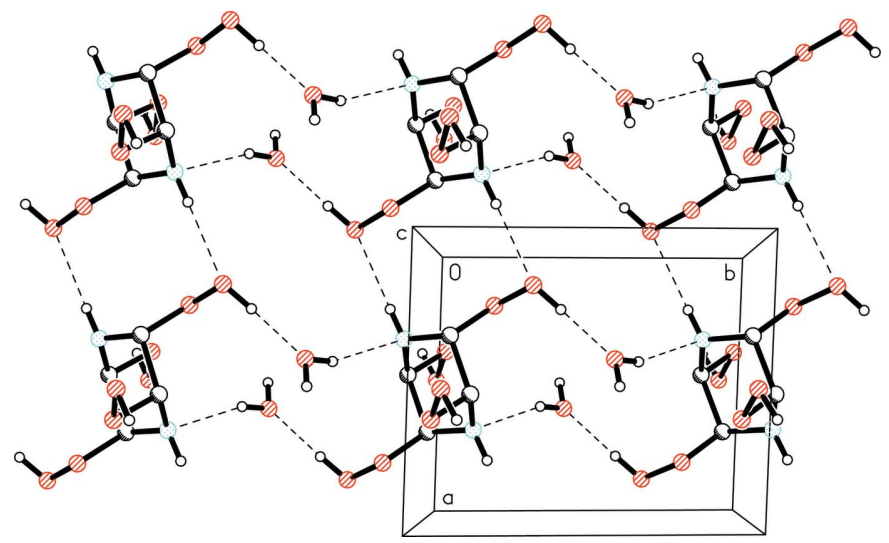

Figure 2

The hydrogen-bonded (dashed lines) layer in (I) perpendicular to the $c$ axis. $\mathrm{H}$ atoms not involved in hydrogen bonds have been omitted for clarity.

first example of a molecular structure containing four $\mathrm{OOH}$ substituents. To the best of our knowledge, (I) is one of the most rich in oxygen organic molecules.

\section{Experimental}

Pyrazine (99\%) and 50\% hydrogen peroxide were purchased from Aldrich. Pyrazine $(0.03 \mathrm{~g})$ was dissolved in approximately $1 \mathrm{ml}$ of $50 \% \mathrm{H}_{2} \mathrm{O}_{2}$. This solution was stored in a freezer at $255 \mathrm{~K}$. After six months, several tiny crystals were found on the wall of a sample bottle. The amount of crystalline material was not enough to investigate it with usual spectroscopic methods. In order to analyse the mother liquor by NMR, it was evaporated in vacuum and the residual oil was dissolved in $\mathrm{D}_{2} \mathrm{O}$. Unfortunately, the recorded ${ }^{1} \mathrm{H}$ and ${ }^{13} \mathrm{C}$ spectra of this complex mixture were non-interpretable.

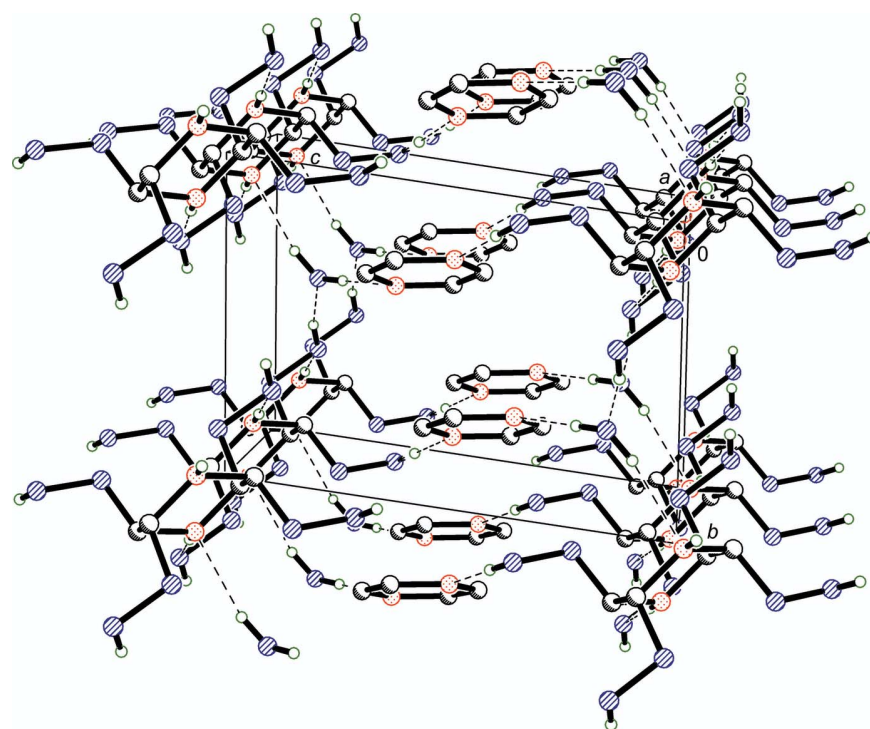

Figure 3

The crystal packing of (I), viewed approximately along the $a$ axis. Hydrogen bonds are shown as dashed lines. $\mathrm{H}$ atoms not involved in hydrogen bonds have been omitted for clarity.

\section{Crystal data}

$\mathrm{C}_{4} \mathrm{H}_{10} \mathrm{~N}_{2} \mathrm{O}_{8} \cdot 2 \mathrm{C}_{4} \mathrm{H}_{4} \mathrm{~N}_{2} \cdot 2 \mathrm{H}_{2} \mathrm{O}$

$M_{r}=410.36$

Triclinic, $P \overline{1}$

$a=6.1538(6) \AA$

$b=7.3047(8) \AA$

$c=10.3364(12) \AA$

$\alpha=97.729$ (3)

$\beta=95.974(4)^{\circ}$

$\gamma=91.417(3)^{\circ}$

$V=457.56(9) \AA^{3}$

$Z=1$

$D_{x}=1.489 \mathrm{Mg} \mathrm{m}^{-3}$

Mo $K \alpha$ radiation

$\mu=0.13 \mathrm{~mm}^{-1}$

$T=120(2) \mathrm{K}$

Needle, colourless

$0.25 \times 0.04 \times 0.03 \mathrm{~mm}$

\section{Data collection}

Bruker SMART $6 \mathrm{~K}$ diffractometer $\omega$ scans

Absorption correction: multi-scan (SADABS; Sheldrick, 1997)

$T_{\min }=0.968, T_{\max }=0.996$

2645 measured reflections 2162 independent reflections 1710 reflections with $I>2 \sigma(I)$ $R_{\text {int }}=0.012$

$\theta_{\text {max }}=28.0^{\circ}$

\section{Refinement}

Refinement on $F^{2}$

$R\left[F^{2}>2 \sigma\left(F^{2}\right)\right]=0.037$

$w R\left(F^{2}\right)=0.090$

$S=0.99$

2162 reflections

170 parameters

All $\mathrm{H}$-atom parameters refined

$$
\begin{gathered}
w=1 /\left[\sigma^{2}\left(F_{\mathrm{o}}{ }^{2}\right)+(0.0248 P)^{2}\right. \\
\quad+0.2345 P] \\
\text { where } P=\left(F_{\mathrm{o}}{ }^{2}+2 F_{\mathrm{c}}^{2}\right) / 3 \\
(\Delta / \sigma)_{\max }<0.001 \\
\Delta \rho_{\max }=0.31 \mathrm{e} \AA^{-3} \\
\Delta \rho_{\min }=-0.23 \mathrm{e}^{-3}
\end{gathered}
$$

Table 1

Hydrogen-bond geometry $\left(\AA,^{\circ}\right)$.

\begin{tabular}{lllll}
\hline$D-\mathrm{H} \cdots A$ & $D-\mathrm{H}$ & $\mathrm{H} \cdots A$ & $D \cdots A$ & $D-\mathrm{H} \cdots A$ \\
\hline $\mathrm{O} 4-\mathrm{H} 41 \cdots \mathrm{N} 1^{\text {i }}$ & $0.88(2)$ & $1.88(2)$ & $2.7573(18)$ & $176.2(18)$ \\
$\mathrm{O}^{\mathrm{ii}}-\mathrm{H} 31 \cdots \mathrm{O} 5^{\text {ii }}$ & $0.88(2)$ & $1.74(2)$ & $2.6068(16)$ & $168.2(19)$ \\
$\mathrm{N}^{-\mathrm{H} 32 \cdots \mathrm{O} 3}{ }^{\text {ii }}$ & $0.83(2)$ & $2.17(2)$ & $2.9985(16)$ & $178.1(17)$ \\
$\mathrm{O}^{2}-\mathrm{H} 51 \cdots \mathrm{N} 2$ & $0.88(3)$ & $1.97(3)$ & $2.8483(19)$ & $177(2)$ \\
$\mathrm{O}^{-\mathrm{H}} 52 \cdots \mathrm{N} 3^{\text {iv }}$ & $0.86(3)$ & $2.14(3)$ & $2.9648(18)$ & $160(2)$ \\
\hline
\end{tabular}

Symmetry codes: (i) $x-1, y, z$; (ii) $x, y+1, z+1$; (iii) $-x,-y+2,-z+2$; (iv) $x, y, z-1$. 
All $\mathrm{H}$ atoms were located in a difference Fourier map and refined isotropically.

Data collection: SMART (Bruker, 2001); cell refinement: SAINT (Bruker, 2001); data reduction: $S A I N T$; program(s) used to solve structure: SHELXS97 (Sheldrick, 1997); program(s) used to refine structure: SHELXL97 (Sheldrick, 1997); molecular graphics: SHELXTL-Plus (Bruker, 2000); software used to prepare material for publication: SHELXTL-Plus.

AVC is grateful to the Russian Science Support Foundation.

\section{References}

Allen, F. H. (2002). Acta Cryst. B58, 380-388.

Bruker (2000). SHELXTL-Plus. Bruker AXS Inc., Madison, Wisconsin, USA. Bruker (2001). SMART and SAINT. Bruker AXS Inc., Madison, Wisconsin, USA.

Churakov, A. V., Prikhodchenko, P. V. \& Howard, J. A. K. (2005). CrystEngComm, 7, 664-669.

Churakov, A. V., Prikhodchenko, P. V., Kuz'mina, L. G. \& Howard, J. A. K. (2004). Chem. Listy, 98, s43-s44.

Marwah, P., Marwah, A. \& Lardy, H. A. (2004). Green Chem. 6, 570-577.

Sheldrick, G. M. (1997). SADABS, SHELXS97 and SHELXL97. University of Göttingen, Germany. 


\title{
supporting information
}

Acta Cryst. (2006). E62, o2265-o2267 [https://doi.org/10.1107/S1600536806016485]

\section{The first molecular structure containing four hydroperoxo groups:} piperazine-2,3,5,6-tetrayl tetrahydroperoxide pyrazine disolvate dihydrate

\author{
Andrei V. Churakov, Lyudmila G. Kuz'mina, Petr V. Prikhodchenko and Judith A. K. Howard
}

piperazine-2,3,5,6-tetrayl tetrahydroperoxide pyrazine disolvate dihydrate

\section{Crystal data}

$\mathrm{C}_{4} \mathrm{H}_{10} \mathrm{~N}_{2} \mathrm{O}_{8} \cdot 2 \mathrm{C}_{4} \mathrm{H}_{4} \mathrm{~N}_{2} \cdot 2 \mathrm{H}_{2} \mathrm{O}$

$M_{r}=410.36$

Triclinic, $P \overline{1}$

Hall symbol: -P 1

$a=6.1538(6) \AA$

$b=7.3047(8) \AA$

$c=10.3364(12) \AA$

$\alpha=97.729(3)^{\circ}$

$\beta=95.974(4)^{\circ}$

$\gamma=91.417(3)^{\circ}$

$V=457.56(9) \AA^{3}$

\section{Data collection}

Bruker SMART 6K diffractometer

Radiation source: fine-focus sealed tube

Graphite monochromator

$\omega$ scans

Absorption correction: multi-scan

(SADABS; Sheldrick, 1997)

$T_{\min }=0.968, T_{\max }=0.996$

\section{Refinement}

Refinement on $F^{2}$

Least-squares matrix: full

$R\left[F^{2}>2 \sigma\left(F^{2}\right)\right]=0.037$

$w R\left(F^{2}\right)=0.090$

$S=0.99$

2162 reflections

170 parameters

0 restraints

Primary atom site location: structure-invariant direct methods
$Z=1$

$F(000)=216$

$D_{\mathrm{x}}=1.489 \mathrm{Mg} \mathrm{m}^{-3}$

Mo $K \alpha$ radiation, $\lambda=0.71073 \AA$

Cell parameters from 1131 reflections

$\theta=2.8-30.5^{\circ}$

$\mu=0.13 \mathrm{~mm}^{-1}$

$T=120 \mathrm{~K}$

Needle, colourless

$0.25 \times 0.04 \times 0.03 \mathrm{~mm}$

2645 measured reflections

2162 independent reflections

1710 reflections with $I>2 \sigma(I)$

$R_{\text {int }}=0.012$

$\theta_{\max }=28.0^{\circ}, \theta_{\min }=2.0^{\circ}$

$h=-8 \rightarrow 7$

$k=-9 \rightarrow 9$

$l=-13 \rightarrow 11$

Secondary atom site location: difference Fourier map

Hydrogen site location: difference Fourier map

All $\mathrm{H}$-atom parameters refined

$w=1 /\left[\sigma^{2}\left(F_{\mathrm{o}}^{2}\right)+(0.0248 P)^{2}+0.2345 P\right]$

where $P=\left(F_{\mathrm{o}}^{2}+2 F_{\mathrm{c}}^{2}\right) / 3$

$(\Delta / \sigma)_{\max }<0.001$

$\Delta \rho_{\max }=0.31 \mathrm{e} \AA^{-3}$

$\Delta \rho_{\min }=-0.23$ e $\AA^{-3}$ 


\section{Special details}

Geometry. All e.s.d.'s (except the e.s.d. in the dihedral angle between two 1.s. planes) are estimated using the full covariance matrix. The cell e.s.d.'s are taken into account individually in the estimation of e.s.d.'s in distances, angles and torsion angles; correlations between e.s.d.'s in cell parameters are only used when they are defined by crystal symmetry. An approximate (isotropic) treatment of cell e.s.d.'s is used for estimating e.s.d.'s involving 1.s. planes.

Refinement. Refinement of $F^{2}$ against ALL reflections. The weighted $R$-factor $w R$ and goodness of fit $S$ are based on $F^{2}$, conventional $R$-factors $R$ are based on $F$, with $F$ set to zero for negative $F^{2}$. The threshold expression of $F^{2}>\sigma\left(F^{2}\right)$ is used only for calculating $R$-factors (gt) etc. and is not relevant to the choice of reflections for refinement. $R$-factors based on $F^{2}$ are statistically about twice as large as those based on $F$, and $R$ - factors based on ALL data will be even larger.

Fractional atomic coordinates and isotropic or equivalent isotropic displacement parameters $\left(\AA^{2}\right)$

\begin{tabular}{|c|c|c|c|c|}
\hline & $x$ & $y$ & $z$ & $U_{\text {iso }} * / U_{\text {eq }}$ \\
\hline N1 & $1.1231(2)$ & $0.79178(18)$ & $0.50775(13)$ & $0.0261(3)$ \\
\hline $\mathrm{N} 2$ & $0.7499(2)$ & $0.63944(18)$ & $0.35086(14)$ & $0.0290(3)$ \\
\hline $\mathrm{C} 1$ & $0.9426(3)$ & $0.7464(2)$ & $0.55999(16)$ & $0.0281(3)$ \\
\hline $\mathrm{C} 2$ & $0.7583(3)$ & $0.6716(2)$ & $0.48149(18)$ & $0.0299(4)$ \\
\hline $\mathrm{C} 3$ & $1.1158(3)$ & $0.7607(2)$ & $0.37735(15)$ & $0.0256(3)$ \\
\hline $\mathrm{C} 4$ & $0.9304(3)$ & $0.6850(2)$ & $0.29966(16)$ & $0.0272(3)$ \\
\hline N3 & 0.32175 & $0.87876(16)$ & $0.97556(12)$ & $0.0182(3)$ \\
\hline $\mathrm{C} 5$ & $0.3104(2)$ & $1.03857(19)$ & $1.07043(14)$ & $0.0176(3)$ \\
\hline C6 & $0.4605(2)$ & $0.88510(19)$ & $0.87366(14)$ & $0.0182(3)$ \\
\hline $\mathrm{O} 1$ & $0.36537(16)$ & $1.00211(14)$ & $0.78281(10)$ & $0.0218(2)$ \\
\hline $\mathrm{O} 2$ & $0.19076(15)$ & $1.17748(13)$ & $1.00797(10)$ & $0.0200(2)$ \\
\hline $\mathrm{O} 3$ & $0.11802(16)$ & $1.30694(14)$ & $1.11516(11)$ & $0.0228(2)$ \\
\hline $\mathrm{O} 4$ & $0.47963(18)$ & $0.96406(16)$ & $0.66468(11)$ & $0.0265(3)$ \\
\hline O5 & $0.4042(2)$ & $0.58225(17)$ & $0.14218(13)$ & $0.0325(3)$ \\
\hline $\mathrm{H} 1$ & $0.940(3)$ & $0.768(3)$ & $0.651(2)$ & $0.033(5)^{*}$ \\
\hline $\mathrm{H} 2$ & $0.628(3)$ & $0.643(3)$ & $0.519(2)$ & $0.040(5)^{*}$ \\
\hline $\mathrm{H} 3$ & $1.240(3)$ & $0.794(2)$ & $0.3417(18)$ & $0.027(5)^{*}$ \\
\hline $\mathrm{H} 4$ & $0.929(3)$ & $0.668(2)$ & $0.2075(19)$ & $0.029(5)^{*}$ \\
\hline H51 & $0.508(4)$ & $0.600(4)$ & $0.209(3)$ & $0.068(8)^{*}$ \\
\hline $\mathrm{H} 52$ & $0.412(4)$ & $0.661(4)$ & $0.088(3)$ & $0.061(7)^{*}$ \\
\hline H5 & $0.230(3)$ & $1.008(2)$ & $1.1425(16)$ & $0.017(4)^{*}$ \\
\hline H6 & $0.469(2)$ & $0.764(2)$ & $0.8258(15)$ & $0.012(4)^{*}$ \\
\hline $\mathrm{H} 32$ & $0.202(3)$ & $0.826(3)$ & $0.9490(18)$ & $0.026(5)^{*}$ \\
\hline $\mathrm{H} 31$ & $0.211(3)$ & $1.400(3)$ & $1.1126(19)$ & $0.034(5)^{*}$ \\
\hline $\mathrm{H} 41$ & $0.369(3)$ & $0.907(3)$ & $0.612(2)$ & $0.034 *$ \\
\hline
\end{tabular}

Atomic displacement parameters $\left(\AA^{2}\right)$

\begin{tabular}{lllllll}
\hline & $U^{11}$ & $U^{22}$ & $U^{33}$ & $U^{12}$ & $U^{13}$ & $U^{23}$ \\
\hline N1 & $0.0255(7)$ & $0.0265(7)$ & $0.0251(7)$ & $0.0008(5)$ & $-0.0012(5)$ & $0.0022(5)$ \\
N2 & $0.0236(7)$ & $0.0254(7)$ & $0.0367(8)$ & $0.0001(5)$ & $-0.0034(6)$ & $0.0042(6)$ \\
C1 & $0.0340(9)$ & $0.0272(8)$ & $0.0236(8)$ & $0.0036(6)$ & $0.0055(6)$ & $0.0028(6)$ \\
C2 & $0.0250(8)$ & $0.0265(8)$ & $0.0399(9)$ & $0.0009(6)$ & $0.0086(7)$ & $0.0064(7)$ \\
C3 & $0.0248(8)$ & $0.0268(8)$ & $0.0255(8)$ & $-0.0005(6)$ & $0.0023(6)$ & $0.0050(6)$ \\
C4 & $0.0304(8)$ & $0.0271(8)$ & $0.0234(8)$ & $0.0025(6)$ & $-0.0017(6)$ & $0.0043(6)$ \\
N3 & $0.0118(5)$ & $0.0184(6)$ & $0.0227(6)$ & $-0.0051(4)$ & $-0.0016(4)$ & $0.0009(5)$
\end{tabular}




\begin{tabular}{lllllll} 
C5 & $0.0131(6)$ & $0.0187(6)$ & $0.0204(7)$ & $-0.0018(5)$ & $-0.0004(5)$ & $0.0030(5)$ \\
C6 & $0.0150(6)$ & $0.0171(6)$ & $0.0211(7)$ & $-0.0023(5)$ & $-0.0014(5)$ & $0.0008(5)$ \\
O1 & $0.0198(5)$ & $0.0249(5)$ & $0.0203(5)$ & $0.0018(4)$ & $-0.0001(4)$ & $0.0034(4)$ \\
O2 & $0.0163(5)$ & $0.0197(5)$ & $0.0228(5)$ & $0.0014(4)$ & $-0.0007(4)$ & $0.0007(4)$ \\
O3 & $0.0166(5)$ & $0.0204(5)$ & $0.0303(6)$ & $-0.0018(4)$ & $0.0050(4)$ & $-0.0025(4)$ \\
O4 & $0.0209(5)$ & $0.0369(6)$ & $0.0208(5)$ & $-0.0035(5)$ & $0.0015(4)$ & $0.0024(4)$ \\
O5 & $0.0359(7)$ & $0.0268(6)$ & $0.0327(7)$ & $-0.0132(5)$ & $-0.0085(5)$ & $0.0082(5)$ \\
\hline
\end{tabular}

Geometric parameters $\left(\AA,{ }^{\circ}\right)$

\begin{tabular}{|c|c|c|c|}
\hline $\mathrm{N} 1-\mathrm{C} 3$ & $1.332(2)$ & $\mathrm{C} 5-\mathrm{O} 2$ & $1.4502(16)$ \\
\hline $\mathrm{N} 1-\mathrm{C} 1$ & $1.338(2)$ & $\mathrm{C} 5-\mathrm{C}^{\mathrm{i}}$ & $1.5286(19)$ \\
\hline $\mathrm{N} 2-\mathrm{C} 4$ & $1.333(2)$ & $\mathrm{C} 5-\mathrm{H} 5$ & $0.984(16)$ \\
\hline $\mathrm{N} 2-\mathrm{C} 2$ & $1.334(2)$ & $\mathrm{C} 6-\mathrm{O} 1$ & $1.4427(17)$ \\
\hline $\mathrm{C} 1-\mathrm{C} 2$ & $1.379(2)$ & $\mathrm{C} 6-\mathrm{C}^{\mathrm{i}}$ & $1.5286(19)$ \\
\hline $\mathrm{C} 1-\mathrm{H} 1$ & $0.932(19)$ & C6- 66 & $0.958(16)$ \\
\hline $\mathrm{C} 2-\mathrm{H} 2$ & $0.95(2)$ & $\mathrm{O} 1-\mathrm{O} 4$ & $1.4695(14)$ \\
\hline $\mathrm{C} 3-\mathrm{C} 4$ & $1.382(2)$ & $\mathrm{O} 2-\mathrm{O} 3$ & $1.4713(14)$ \\
\hline $\mathrm{C} 3-\mathrm{H} 3$ & $0.924(18)$ & $\mathrm{O} 3-\mathrm{H} 31$ & $0.88(2)$ \\
\hline $\mathrm{C} 4-\mathrm{H} 4$ & $0.943(19)$ & $\mathrm{O} 4-\mathrm{H} 41$ & $0.88(2)$ \\
\hline $\mathrm{N} 3-\mathrm{C} 5$ & $1.4281(18)$ & $\mathrm{O} 5-\mathrm{H} 51$ & $0.88(3)$ \\
\hline $\mathrm{N} 3-\mathrm{C} 6$ & $1.4279(18)$ & $\mathrm{O} 5-\mathrm{H} 52$ & $0.86(3)$ \\
\hline N3-H32 & $0.83(2)$ & & \\
\hline $\mathrm{C} 3-\mathrm{N} 1-\mathrm{C} 1$ & $116.91(14)$ & $\mathrm{N} 3-\mathrm{C} 5-\mathrm{O} 2$ & $108.69(11)$ \\
\hline $\mathrm{C} 4-\mathrm{N} 2-\mathrm{C} 2$ & $116.13(14)$ & $\mathrm{N} 3-\mathrm{C} 5-\mathrm{C} 6^{\mathrm{i}}$ & $110.81(11)$ \\
\hline $\mathrm{N} 1-\mathrm{C} 1-\mathrm{C} 2$ & $121.06(15)$ & $\mathrm{O} 2-\mathrm{C} 5-\mathrm{C} 6^{\mathrm{i}}$ & $110.11(11)$ \\
\hline $\mathrm{N} 1-\mathrm{C} 1-\mathrm{H} 1$ & $119.6(12)$ & $\mathrm{N} 3-\mathrm{C} 5-\mathrm{H} 5$ & $110.3(10)$ \\
\hline $\mathrm{C} 2-\mathrm{C} 1-\mathrm{H} 1$ & $119.4(12)$ & $\mathrm{O} 2-\mathrm{C} 5-\mathrm{H} 5$ & $107.5(9)$ \\
\hline $\mathrm{N} 2-\mathrm{C} 2-\mathrm{C} 1$ & $122.40(15)$ & $\mathrm{C} 6-\mathrm{C} 5-\mathrm{H} 5$ & $109.3(10)$ \\
\hline $\mathrm{N} 2-\mathrm{C} 2-\mathrm{H} 2$ & $116.8(13)$ & $\mathrm{N} 3-\mathrm{C} 6-\mathrm{O} 1$ & $108.62(11)$ \\
\hline $\mathrm{C} 1-\mathrm{C} 2-\mathrm{H} 2$ & $120.8(13)$ & $\mathrm{N} 3-\mathrm{C} 6-\mathrm{C} 5^{\mathrm{i}}$ & $111.10(12)$ \\
\hline $\mathrm{N} 1-\mathrm{C} 3-\mathrm{C} 4$ & $121.54(15)$ & $\mathrm{O} 1-\mathrm{C} 6-\mathrm{C} 5^{\mathrm{i}}$ & $109.50(11)$ \\
\hline $\mathrm{N} 1-\mathrm{C} 3-\mathrm{H} 3$ & $116.6(12)$ & $\mathrm{N} 3-\mathrm{C} 6-\mathrm{H} 6$ & $110.2(9)$ \\
\hline $\mathrm{C} 4-\mathrm{C} 3-\mathrm{H} 3$ & $121.9(12)$ & $\mathrm{O} 1-\mathrm{C} 6-\mathrm{H} 6$ & $107.5(9)$ \\
\hline $\mathrm{N} 2-\mathrm{C} 4-\mathrm{C} 3$ & $121.95(15)$ & $\mathrm{C} 55^{\mathrm{i}}-\mathrm{C} 6-\mathrm{H} 6$ & $109.8(9)$ \\
\hline $\mathrm{N} 2-\mathrm{C} 4-\mathrm{H} 4$ & $118.5(11)$ & $\mathrm{C} 6-\mathrm{O} 1-\mathrm{O} 4$ & $105.82(9)$ \\
\hline $\mathrm{C} 3-\mathrm{C} 4-\mathrm{H} 4$ & $119.5(11)$ & $\mathrm{C} 5-\mathrm{O} 2-\mathrm{O} 3$ & $106.00(9)$ \\
\hline $\mathrm{C} 5-\mathrm{N} 3-\mathrm{C} 6$ & $119.31(11)$ & $\mathrm{O} 2-\mathrm{O} 3-\mathrm{H} 31$ & $98.2(12)$ \\
\hline $\mathrm{C} 5-\mathrm{N} 3-\mathrm{H} 32$ & $113.8(12)$ & $\mathrm{O} 1-\mathrm{O} 4-\mathrm{H} 41$ & $97.7(12)$ \\
\hline $\mathrm{C} 6-\mathrm{N} 3-\mathrm{H} 32$ & $112.9(12)$ & $\mathrm{H} 51-\mathrm{O} 5-\mathrm{H} 52$ & $113(2)$ \\
\hline $\mathrm{C} 3-\mathrm{N} 1-\mathrm{C} 1-\mathrm{C} 2$ & $-0.2(2)$ & $\mathrm{C} 6-\mathrm{N} 3-\mathrm{C} 5-\mathrm{C} 6^{\mathrm{i}}$ & $-49.07(18)$ \\
\hline $\mathrm{C} 4-\mathrm{N} 2-\mathrm{C} 2-\mathrm{C} 1$ & $-0.5(2)$ & $\mathrm{C} 5-\mathrm{N} 3-\mathrm{C} 6-\mathrm{O} 1$ & $-71.31(15)$ \\
\hline $\mathrm{N} 1-\mathrm{C} 1-\mathrm{C} 2-\mathrm{N} 2$ & $0.5(3)$ & $\mathrm{C} 5-\mathrm{N} 3-\mathrm{C} 6-\mathrm{C} 5^{\mathrm{i}}$ & $49.20(18)$ \\
\hline $\mathrm{C} 1-\mathrm{N} 1-\mathrm{C} 3-\mathrm{C} 4$ & $0.0(2)$ & $\mathrm{N} 3-\mathrm{C} 6-\mathrm{O} 1-\mathrm{O} 4$ & $-165.20(10)$ \\
\hline $\mathrm{C} 2-\mathrm{N} 2-\mathrm{C} 4-\mathrm{C} 3$ & $0.2(2)$ & $\mathrm{C} 5^{\mathrm{i}}-\mathrm{C} 6-\mathrm{O} 1-\mathrm{O} 4$ & $73.31(12)$ \\
\hline
\end{tabular}




\begin{tabular}{llll}
$\mathrm{N} 1-\mathrm{C} 3-\mathrm{C} 4-\mathrm{N} 2$ & $0.0(2)$ & $\mathrm{N} 3-\mathrm{C} 5-\mathrm{O} 2-\mathrm{O} 3$ & $160.69(10)$ \\
$\mathrm{C} 6-\mathrm{N} 3-\mathrm{C} 5-\mathrm{O} 2$ & $72.06(15)$ & $\mathrm{C} 6-\mathrm{C} 5-\mathrm{O} 2-\mathrm{O} 3$ & $-77.76(12)$ \\
\hline
\end{tabular}

Symmetry code: (i) $-x+1,-y+2,-z+2$.

Hydrogen-bond geometry $\left(A,{ }^{\circ}\right)$

\begin{tabular}{lllll}
\hline$D-\mathrm{H} \cdots A$ & $D-\mathrm{H}$ & $\mathrm{H} \cdots A$ & $D \cdots A$ & $D-\mathrm{H} \cdots A$ \\
\hline $\mathrm{O} 4-\mathrm{H} 41 \cdots \mathrm{N} 1^{\mathrm{ii}}$ & $0.88(2)$ & $1.88(2)$ & $2.7573(18)$ & $176.2(18)$ \\
$\mathrm{O} 3-\mathrm{H} 31 \cdots 5^{\mathrm{iii}}$ & $0.88(2)$ & $1.74(2)$ & $2.6068(16)$ & $168.2(19)$ \\
$\mathrm{N} 3-\mathrm{H} 32 \cdots \mathrm{O} 3^{\text {iv }}$ & $0.83(2)$ & $2.17(2)$ & $2.9985(16)$ & $178.1(17)$ \\
$\mathrm{O} 5-\mathrm{H} 51 \cdots \mathrm{N} 2$ & $0.88(3)$ & $1.97(3)$ & $2.8483(19)$ & $177(2)$ \\
$\mathrm{O} 5-\mathrm{H} 52 \cdots \mathrm{N} 3^{v}$ & $0.86(3)$ & $2.14(3)$ & $2.9648(18)$ & $160(2)$
\end{tabular}

Symmetry codes: (ii) $x-1, y, z$; (iii) $x, y+1, z+1$; (iv) $-x,-y+2,-z+2$; (v) $x, y, z-1$. 